\title{
OCCLUDED AREA REMOVING FROM HANDHELD LASER SCANNER DATA DURING 3D BUILDING MODELLING
}

\author{
F. Sadeghi ${ }^{1, *}$, H. Arefi ${ }^{2}$ \\ ${ }^{1}$ Fatemehsadeghi@alumni.ut.ac.ir \\ ${ }^{2}$ School of Surveying and Geospatial Engineering, University of Tehran, Tehran, Iran (hosein.arefi@gmail.com)
}

KEYWORDS: 3D modelling, Occluded area, RanSAC, Zeb1, Point cloud

\begin{abstract}
:
3D building modelling has been turned to be one of the most interesting and hottest subjects in photogrammetry in last two decades, and it seems that photogrammetry provides the only economic means to acquire truly 3D city-data. Most of the researches proposed methods for 3d building modelling in LoD2 using aerial images and LIDAR data and the produced models will be enriched by oblique images, therefore there is always a demand for a user to interpret the façade or in other manual building reconstruction process the operator should draw boundaries to represent the building model and the process will be too time-consuming for $3 \mathrm{~d}$ modelling for a whole city. Creating building facade models for a whole city requires considerable work, therefore for decades, much research has been dedicated to the automation of this reconstruction process. Nowadays researchers attempt to recommend a new method which is flexible to model hug variety of buildings and has a solution for several challenges such as irrelevant objects (pedestrians, trees, traffic signs, etc.), occluded areas and non-homogenous data. Based on various 3d building models applications, namely navigation systems, location-based system, city planning and etc. the demand for adding semantic features (such as windows and doors) is increasing and becoming more essential, therefore simple blocks as the representation of $3 \mathrm{~d}$ buildings aren't sufficient anymore. Therefore 2.5 models which show the façade details using pixel values have been substituted by LoD3 models recently.

The lack of automation in image based approaches can be explained by the difficulties in image interpretation. Specifically, factors like illumination and occlusion can cause considerable confusion for machine understanding and some conditions (relative orientation, feature matching, etc.) need to be accurately determined to transfer image pixels to 3D coordinates. In recent years, terrestrial laser scanning data has been proven as a valuable source for building facade reconstruction. The point density of stationary laser scanning in urban areas can be up to hundreds or thousands of points per square meter, which is high enough for documenting most details on building facades. In comparison with image-based modelling, several steps such as image matching, intersection and resection will be eliminated, while there is no need to image interpret in laser data-based reconstruction approaches, these methods face major challenges such as extracting meaningful structures from a huge amount of data.

This paper presents a data-driven algorithm for façade reconstruction, using a handheld laser scanner, Zebedee. The mentioned device is consisting of $2 \mathrm{~d}$ laser scanner and an inertial measurement unit mounted on one or two springs, it has 270-degree field of view. Its mass is $210 \mathrm{~g}$ which makes it ideal for low measurement and it is maximum range is $30 \mathrm{~m}$. The proposed method was implemented by using the Zebedee point cloud in order to determine the challenges of zeb1 data and ensure that the introduced device can be practical for $3 \mathrm{~d}$ reconstruction.

Due to obstacle existence, operator gross errors while data capturing and façade elements arrangement, there will always be occluded area and shadows in produced data. Occluded area cause tribulation in machine understanding and problems for automatic reconstruction algorithms. The proposed method represents a new way to detect occluded area and remove the artificial objects which are produced by them. The $3 \mathrm{~d}$ point cloud is used to cover all façade elements and details, also image matching and producing 3-dimensional data steps will be omitted from the process.

The proposed workflow is indicated in figure 1. Most researches such as road, building or other objects detection and reconstruction put ground points detection in priority in order to decrease data volume and processing time, so as a pre-processing step, point cloud is classified into two separate groups (non-ground and ground points).
\end{abstract}

\section{INTRODUCTION}

Images were the first data which were utilized for building reconstruction since they provide optical information and also they are easy to acquire and additionally, they will cost cheap if the camera is non-metric. Despite the fact that images are easy to interpret and perfect for visualization, there are a few methods to extract building structures automatically and factors like illumination and occlusion make confusion for machine understanding and procedures like feature matching and other steps are so time-consuming. In recent years, point cloud data has been known as a valuable source for building reconstruction. The point density of stationary laser scanning in urban areas can be up to hundreds or thousands of points per square meter, which is high enough for documenting most details on building facades. Using MLS or handheld laser scanner cause omit stationing step in data capturing process.
(Pu et al. 2009) The 3d data is non-homogenous and there is always occluded area because of irrelevant objects (trees, pedestrians, cars, traffic signs, poles) and façade structure. In the following, some of the existing approaches are classified according to the data and introduced:

\subsection{Related works}

(Penrad et al. 2017) uses a set of overlapping images which were generated via stereopolis system, to generate mesh models automatically. In this process based on selected building, images which have whole or partially part of the building will be chosen for the rest of the process, for each viewpoint, point cloud will be produced and filtered out. In the final step, a mesh model will be created from the reliable $3 \mathrm{~d}$ point cloud. 
(Frueh 2017) used digital images and terrestrial laser points as input to produce mesh models. At first Laser point clouds are used to generate depth images, by producing a histogram and analyzing it, unrelated objects and facades are separated, façade 's points will be used to produce TIN mesh models, finally, the texture will be mapped from images.

(Lindequist Larson 2017) in this method, calibration parameters for all images are estimated and then key points in all images will be detected. In next step, matching will be performed for each two images then position of key points will be determined, in final step a coarse model of building will be represented as a textured mesh, which consists of large planner surface such as roof, wall and etc. reconstruction will be completed by $3 \mathrm{~d}$ position of sharp features in 2 or more than it in images. So the shape of the coarse model will be defined interactively by the user and then textures for every single polygon will be mapped by input images and then building details will be added to the coarse model.

(Pu et al.) Line extraction from images is very accurate, while laser points are more suitable for extracting planar features. a building facade's general structure is established with the planar features extracted from laser data, in the next step, image features are introduced to refine the model details. Therefore, significant line features extracted from images are compared with the initial model's edges, and necessary refinements are made according to the image lines. Textures of different model faces are selected automatically from multiple images to ensure optimal visibility.

(Tutzauer 2017) Used point cloud derived from oblique airborne images and from the mobile mapping system. This work is an extension of grammar-based modeling which has been done by (Becker 2009), this paper represents a new radiometric segmentation approach when geometric accuracy is limited and geometric reconstruction fails. In this method user can choose façade to reconstruct, then points related to the selected façade will be cut, then the coarse building model and related point cloud are considered as input. After performing data-driven reconstruction which concludes geometric segmentation (radiometric reconstruction if it is necessary), façade will be completed by forming façade vocabularies and their interrelations (grammar)

( $\mathrm{Pu}$ et al. 2009) introduced knowledge-based 3D building modelling. This process includes some steps; façade point cloud is segmented into different parts. Based on several properties such as height, position and topologic relations, the segmented parts are recognized and modelled. These properties are interfered from homo sapiens knowledge.

\subsection{Overview of the algorithm}

In this paper, we proposed a data-driven method to reconstruct building modeling by using handheld laser scanner data which can remove the occluded area. The method process has been shown in flowchart figure 1 and details will be discussed in the following sections.

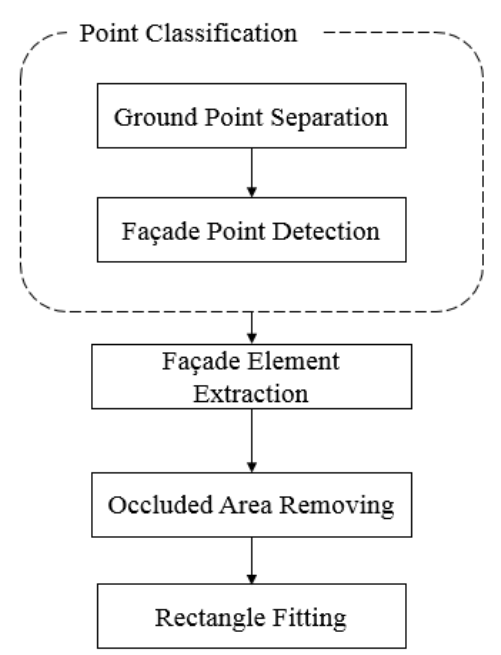

\subsection{Data-set}

Figure 1. Overview of the algorithm

Zebedee (figure 2) has been known as one of the most advanced devices for producing $3 \mathrm{~d}$ point cloud in the last 5 years. Its simplicity in structure, size, and weight make it practical to be used for capturing data. The Zebedee 3D sensor system consists of a 2D laser scanner and an IMU mounted on one or more springs (Sirmacek 2017). As an operator holding the device moves through the environment, the scanner loosely oscillates about the spring, thereby producing rotational motion that converts the laser's inherent $2 \mathrm{D}$ scanning plane into a local $3 \mathrm{D}$ field of view and furthermore, by rotating the spring, the field of view will be increased. The proposed method was implemented by using a handheld laser scanner point cloud in order to determine the challenges of zeb1 data and ensure that the introduced device is practical for $3 \mathrm{~d}$ reconstruction. Zeb1 is capable of capturing about 43200 points per second. The utilized data set is related to hft 2 building in Applied science university campus in Germany. Variation in density is explicitly shown in figure 3 .

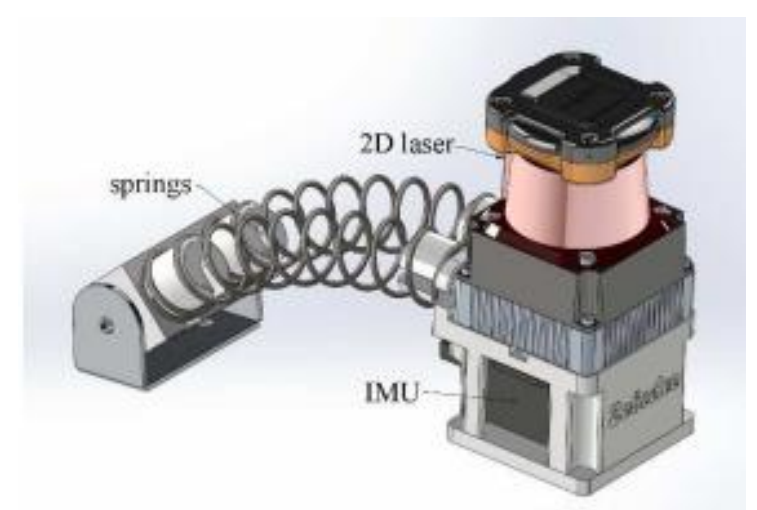

Figure 2. Handheld laser scanner Zebedee 


\section{PROPOSED METHOD}

\subsection{Point classification}

2.1.1 Ground point separation Since ground points form a huge amount of whole data set (as shown in figure 3), so most researches such as road, building or other objects detection and reconstruction put ground points detection in priority in order to decrease data volume and processing time, so as pre-processing step, by using (Axelsson )method, point cloud was classified into two separate groups as ground and non-ground points as shown in figure 4 using LaStools software.

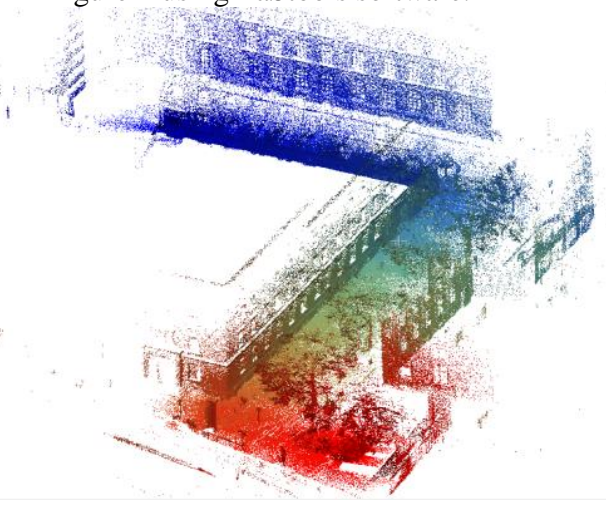

Figure 3. captured point cloud from Zeb1

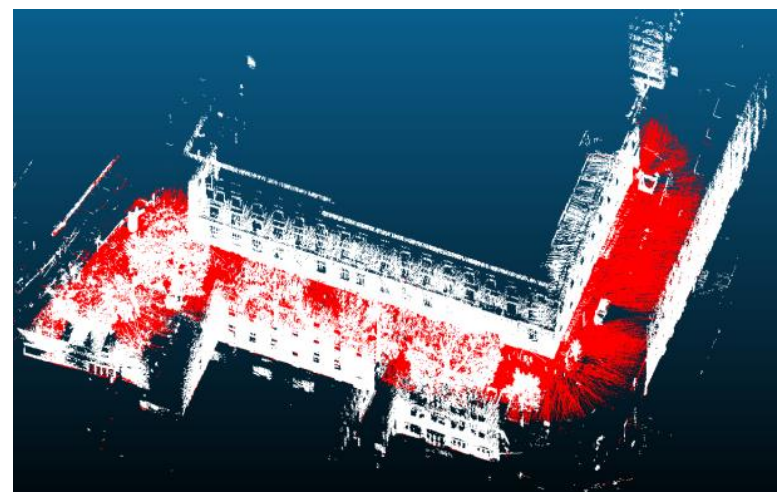

Figure 4. ground and non-ground points which are separated in white and red colors respectively

2.1.2 Façade point detection Non-ground points contain irrelevant objects such as trees, pedestrians, poles, and buildings. Since facades have regular structures and similar patterns, they can be extracted by using geometric properties. So the façade plane must be separated from other objects. For this, the conditional RanSAC (which is introduced by (Schnabel et al. 2007)) is used to detect walls in a way of keeping the windowsill and niches information, detect the major planes and increase the robustness of the method. Then two histograms are implemented in both $\mathrm{y}$ and $\mathrm{x}$-direction on a depth image (figure 5 ) which is produced by gridding façade point in order to cut residual irrelevant objects from two dimensions and cut inqualified data. Figure 6 shows cut qualified data as a result of this step.

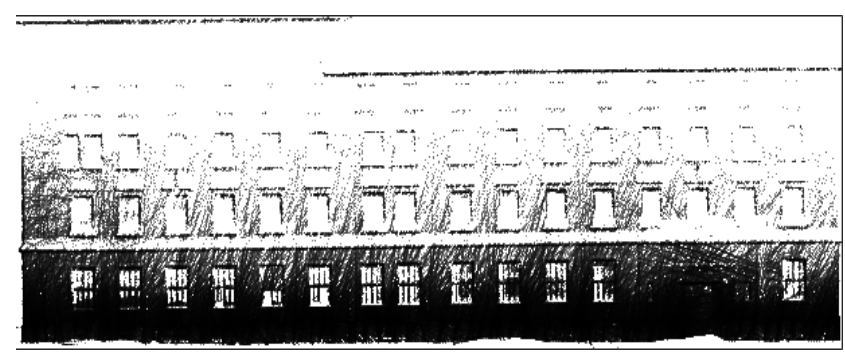

Figure 5. The binary image of façade

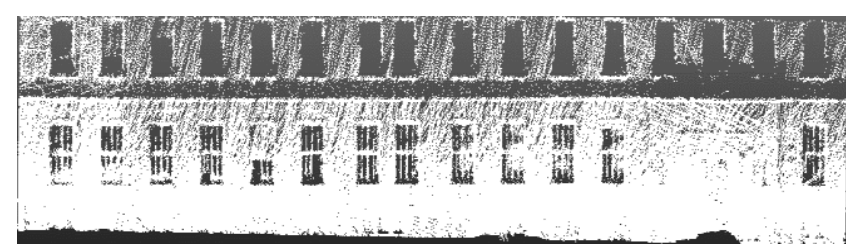

Figure 6. qualified façade points

\subsection{Façade element extraction}

facade plane normally has a depth which contains indentation and protrusion points. Inner points based on the façade structure indicate façade elements information. In order to separate the windows and door, a histogram perpendicular to façade plane is implemented. The histogram has normally three peaks, showing many indentation, main wall and protrusion points, and their positions show depth. Indentation and protrusion indicate niches, windows and door information respectively. Figure 7 shows depth histogram. In this step noises and irrelevant object which are in front of façade are eliminated. The absolute maximum and adjacent relative maximums show wall, façade elements and ledges respectively.

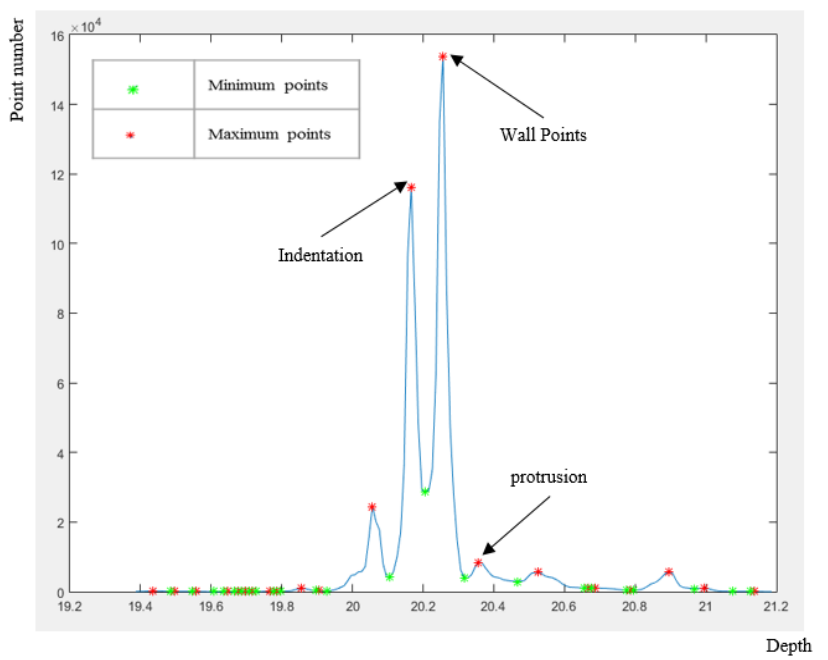

Figure 7. depth histogram

\subsection{Occluded area removing}

Due to 15 to $30 \mathrm{~m}$ range of zeb1, building façade can't be scan completely and there is lack of information for upper parts of the building, furthermore obstacles and protrusion cause shadow and the occluded area in the point cloud. Furthermore, operator mistakes during capturing data and scanning cause low-quality data. Occluded area cause tribulation in machine understanding and problems for automatic reconstruction algorithms. In order to prevent producing artificial elements, a $2 \mathrm{~d}$ low-resolution binary image is produced by gridding (whole) 
façade points. A pixel will get zero value if the related grid element contains point and pixel will be one if the grid element is empty and another binary image is produced using inner façade points which used opposite previous process. Figure 8 and 9 show the binary image related to façade 's inner points and inner points respectively.

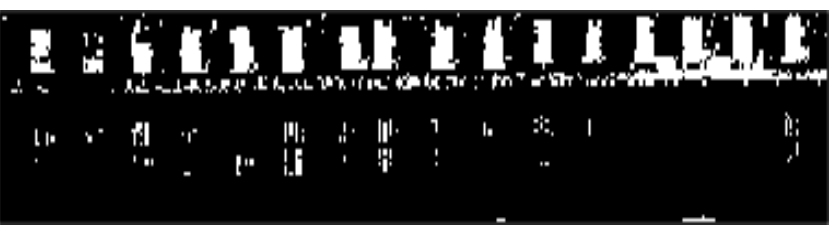

Figure 8. inner point s' binary image (IPBI)

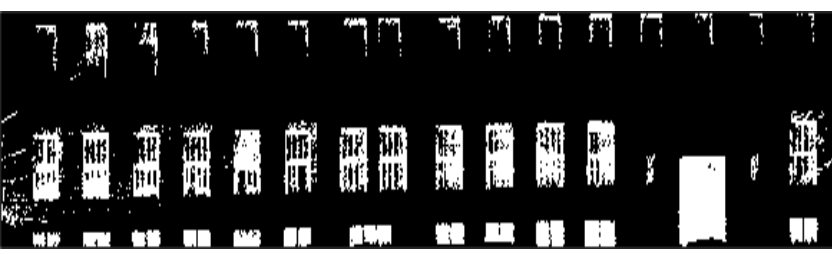

Figure 9. façade point s' binary image (FPBI)

Then connected components in the IPBI ${ }^{1}$ are recognized and if number of each component is less than the threshold (which is determined by the user), it will be considered as noise and will be omitted, otherwise, it will be maintained for rest of process. In order to improve the algorithm and accelerate of process time, every façade element in $\mathrm{FPBI}^{2}$ is considered as a search area in the IPBI and if there is more than one connected component in the search area, the union of them will be added to the previous façade element to improve element structure (Figure 10.).

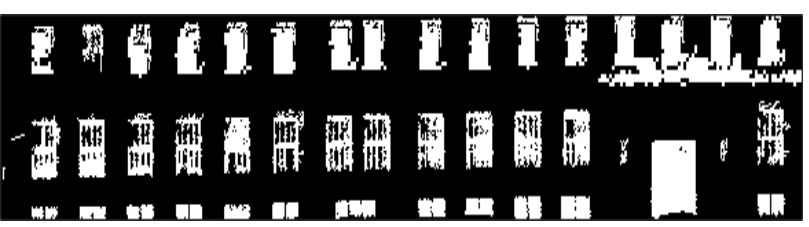

Figure 10. the union of two images (in figure 8 and figure 9) in search areas

By using image processing toolbox, artificial elements and noises will be omitted and façade elements will be modified. Connected components pixels' average determines a threshold. If façade element pixels' number is more than the threshold, it will be considered as a combination of façade element and noise, for removing noise from the element, the morphological operation will be implemented on the element until pixel number is in the optimum range. Finally, the noise will be omitted by considering width to length ratio (from former knowledge of buildings) and the main separated element will be modified using dilation operator as many times as processed by erosion operator in the previous step. Figure 11 shows the result of this step.

\footnotetext{
${ }^{1}$ Inner Point s' Binary Image

2 Façade Point s' Binary Image
}

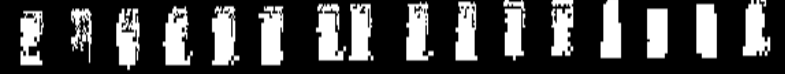

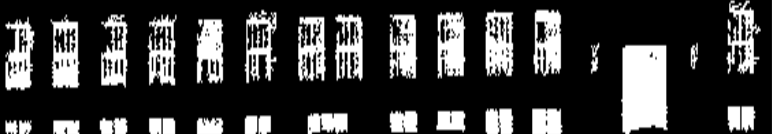

Figure 11. implementing erosion and dilation operations on figure 9

\subsection{Rectangle fitting}

After removing occluded area, each connected component corresponds to an element in façade. By recognizing minimum and maximum row and column of each connected component, a rectangle is fitted to them (Figure 12). According to the transformation relationship of point cloud to binary image twodimensional coordinates are computable, and depth is determined by depth histogram which can be considered as future work.

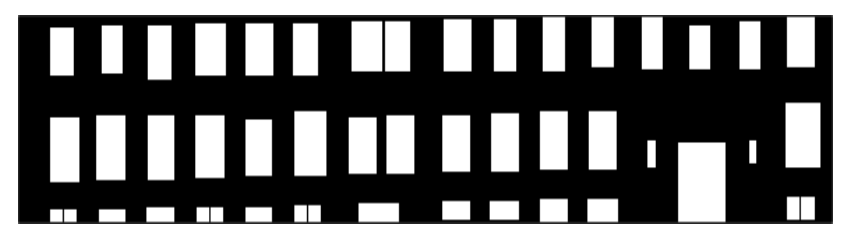

Figure 12. fitted rectangles to connected components

\section{CONCLUSION}

Since there are always challenges during data capturing for the operator and also zeb1 limitation, output data contains defects like the occluded area and always is uncompleted. In this paper, we described our approach for removing artificial objects caused by occluded area, by considering former knowledge about building structure, noisy data can be improved. In comparison with data-driven modelling method in previous work, one-floor elements additionally could be added to the façade model in the reconstruction process.

\section{REFERENCES}

Axelsson, P., 1999, Processing of laser scanner data algorithms and applications, ISPRS Journal of Photogrammetry \& Remote Sensing 54 (2).

Becker, S., 2009. Generation and application of rules for quality dependent facade reconstruction. ISPRS Journal of Photogrammetry and Remote Sensing, 64(6), pp. 640-653.

Frueh, C., Jain, S. and Zakhor, A. 2005. Data processing algorithms for generating textured $3 \mathrm{~d}$ building facade meshes from laser scans and camera images. Int. J. Computer Vision, 61(2), pp. 159-184

Lindequist Larsen, C., 2010, 3D Reconstruction of Buildings from Images with Automatic Facade Refinement, Master's Thesis in Vision, Graphics and Interactive Systems

Penard, L., Paparoditis, N. and Pierrot-Deseilligny, M., 2005, 3d Building Façade Reconstruction under Mesh Model from Multiple Wide Angle Views, International Archived of the Photogrammetry, Remote Sensing and Spatial Information Science (IAPRS), Venice, 36-5 (WG 17, TS9) 
$\mathrm{Pu}, \quad$ S. and Vosselman, G., 2009, Building Façade Reconstruction by Fusing Terrestrial Laser Points and Images, Sensors 2009, 9, 4525-4542

$\mathrm{Pu}, \quad$ S. and Vosselman, G. 2009, Knowledge based reconstruction of building models from terrestrial laser scanning data, ISPRS Journal of Photogrammetry and Remote Sensing, 64 (6), pp. 575-84.

Schnabel, R., Wahl, R., and Klein, R. 2007, Efficient RANSAC for Point-Cloud Shape Detection, Computer Graphics Forum, 26 (2), pp. 214-26

Sirmacek, B., Shen, Y., Lindenbergh, R., Zlatanova, S., Diakite, A., 2016. Comparison of Zeb1 and Leica C10 Indoor Laser Scanning Point Clouds. Int. Ann. Photogrammetry. Remote Sens. Spatial Inf. Sci. III-1, 143-149.

Tutzauer, P. and Haala, N., 2015, Facade reconstruction using geometric and radiometric point cloud information. ISPRS International Archives of the Photogrammetry Remote Sensing and Spatial Information Sciences, 247-252. 